\title{
A temática "Saúde da Mulher" na Revista Brasileira de Obesidade, Nutrição e Emagrecimento: Análise de uma década (2010-2020)
}

The theme "Women's Health" in the Brazilian Journal of Obesity, Nutrition and Weight Loss: Analysis of a decade (2010-2020)

El tema "Salud de la mujer" en la Revista Brasileña de Obesidad, Nutrición y Pérdida de Peso: Análisis de una década (2010-2020)

Recebido: 05/08/2021 | Revisado: 11/08/2021 | Aceito: 16/08/2021 | Publicado: 19/08/2021

Gustavo Baroni Araujo
ORCID: https://orcid.org/0000-0002-3162-7477
Universidade Estadual de Londrina, Brasil
E-mail: gustavobaroni13@ @otmail.com
Thyago de Oliveira Afonso
ORCID: https://orcid.org/0000-0001-7616-9011
Universidade Federal de Pernambuco, Brasil
E-mail: thyago.oafonso@ gmail.com
Joelma Maria dos Santos da Silva Apolinário
ORCID: https://orcid.org/0000-0001-9521-9432
Faculdade Maurício de Nassau, Brasil
E-mail: jo.silva00@ hotmail.com
Mariana Pereira Barbosa Silva
ORCID: https://orcid.org/0000-0003-0852-8099
Universidade Estadual do Piauí, Brasil
E-mail: marianapbsilvaa @ gmail.com
Lorrane de Sousa Barbosa
ORCID: https://orcid.org/0000-0002-3668-2993
Universidade Estadual da Paraíba, Brasil
E-mail: sousablorrane @ gmail.com

\section{Resumo}

O número de mulheres no Brasil é superior ao de homens. A população brasileira é composta por 51,8\% de mulheres e 48,2\% de homens. Com o propósito de atender essa demanda, foram criadas estratégias para garantir atenção à saúde da mulher. O objetivo deste trabalho foi investigar as publicações sobre saúde da mulher na Revista Brasileira de Obesidade, Nutrição e Emagrecimento partindo da relevância da discussão de aspectos nutricionais no que diz respeito ao cuidado integral à saúde da mulher. Trata-se de um estudo de revisão retrospectiva, bibliográfica e exploratória, realizado entre os meses de maio e junho de 2021, com abordagem descritiva, na base de dados "Revista Brasileira de Obesidade, Nutrição e Emagrecimento" através dos descritores/palavras-chave "Saúde da Mulher", "Saúde", "Mulher" e "Mulheres". No total, foram localizados nove artigos. A maior prevalência encontrada foram estudos descritivos, em diversas subáreas do conhecimento, como aspectos epidemiológicos, sociodemográficos da atividade física nas mulheres, tratando modalidades de exercícios físicos em respostas e ajustes metabólicos apresentando atividades preventivas no que se refere aos cuidados nutricionais que ampliam os cuidados em saúde nas mulheres, evitando possíveis prejuízos e adiando o desenvolvimento e agravo de doenças. Conclui-se que a garantia dos cuidados em saúde parte do princípio multidisciplinar e que as questões quanto à alimentação podem interferir em desfechos em saúde, portanto, os profissionais da saúde devem ter conhecimento sobre as características dessa população e para que isso ocorra é importante que exista um conhecimento especifico pautado nas particularidades dessa população.

Palavras-chave: Saúde da mulher; Saúde; Mulheres; Aspectos nutricionais.

\begin{abstract}
The number of women in Brazil is higher than that of men. The Brazilian population is made up of $51.8 \%$ women and $48.2 \%$ men. In order to meet this demand, strategies were created to ensure women's health care. The aim of this study was to investigate the publications on women's health in the Brazilian Journal of Obesity, Nutrition and Weight Loss, starting from the relevance of the discussion of nutritional aspects with regard to comprehensive care for women's health. This is a retrospective, bibliographical and exploratory review study, carried out between May and June 2021, with a descriptive approach, in the database "Brazilian Journal of Obesity, Nutrition and Weight Loss" through the descriptors/key words "Women's Health", "Health", "Women" and "Women". In total, nine articles were found. The
\end{abstract}


highest prevalence found were descriptive studies, in several sub-areas of knowledge, such as epidemiological, sociodemographic aspects of physical activity in women, dealing with physical exercise modalities in responses and metabolic adjustments presenting preventive activities with regard to nutritional care that expand care in health in women, avoiding possible harm and delaying the development and aggravation of diseases. It is concluded that the guarantee of health care is based on the multidisciplinary principle and that food issues can interfere with health outcomes, therefore, health professionals must have knowledge about the characteristics of this population and for this to occur, it is important that there is specific knowledge based on the particularities of this population.

Keywords: Women's health; Health; Women; Nutritional aspects.

\section{Resumen}

El número de mujeres en Brasil es mayor que el de hombres. La población brasileña está compuesta por un $51,8 \%$ de mujeres y un 48,2\% de hombres. Para atender esta demanda, se crearon estrategias para asegurar la atención de la salud de la mujer. El objetivo de este estudio fue investigar las publicaciones sobre salud de la mujer en la Revista Brasileña de Obesidad, Nutrición y Pérdida de Peso, partiendo de la relevancia de la discusión de los aspectos nutricionales en la atención integral a la salud de la mujer. Se trata de un estudio retrospectivo, de revisión bibliográfica y exploratoria, realizado entre mayo y junio de 2021, con enfoque descriptivo, en la base de datos "Revista Brasileña de Obesidad, Nutrición y Pérdida de Peso" a través de los descriptores / palabras clave "Salud de la Mujer", "Salud", "Mujeres" y "Mujeres". En total, se encontraron nueve artículos. Las mayores prevalencias encontradas fueron estudios descriptivos, en várias subáreas del conocimiento, como aspectos epidemiológicos, sociodemográficos de la actividad física en la mujer, que abordan las modalidades de ejercicio físico en las respuestas y ajustes metabólicos que presentan actividades preventivas con respecto al cuidado nutricional que amplían la atención en salud en la mujer, evitando posibles daños y retrasando el desarrollo y agravamiento de las enfermedades. Se concluye que la garantía de la atención en salud se basa en el principio multidisciplinario y que los temas alimentarios pueden interferir con los resultados de salud, por lo tanto, los profesionales de la salud deben tener conocimiento sobre las características de esta población y para que esto ocurra es importante que exista conocimientos específicos basados en las particularidades de esta población.

Palabras clave: Salud de la mujer; Salud; Mujeres; Aspectos nutricionales.

\section{Introdução}

De acordo com os dados da PNAD Contínua (Pesquisa Nacional por Amostra de Domicílios Contínua) 2019, o número de mulheres no Brasil é superior ao de homens. A população brasileira é composta por 51,8\% de mulheres e 48,2\% de homens. Essas informações são relevantes para o mapeamento das diferenças entre os sexos fomentando a criação de políticas públicas para reduzir as disparidades no acesso aos cuidados em saúde (Veras, 2007); (Contatore, Malfitano \& Barros, 2018).

Com o propósito de atender os cuidados em saúde para esse elevado número de mulheres, foram criadas estratégias para garantir atenção à saúde da mulher através de serviços ofertados pelo Sistema Único de Saúde (SUS) oferecendo atendimentos, consultas e medicamentos de forma gratuita para as mulheres de todas as idades (Araujo \& Xavier, 2014).

A implementação da Política Nacional de Atenção Integral à Saúde da Mulher, elaborada em 2004, teve como base o Programa de Atenção Integral de Saúde da Mulher, criado em 1984, a partir do diagnóstico epidemiológico da saúde da mulher no Brasil, surgindo na emergência de atender essa população, baseando-se na promoção da saúde através de melhorias e da manutenção das capacidades funcionais fundamentado pela prevenção, recuperação e reabilitação da saúde de modo que essa população permaneça no meio em que vivem exercendo de forma independente suas funções na sociedade (Brasil, 2004); (Carvalho, 2013); (Cordeiro et al., 2017).

Frente a este cenário, considerando necessidade de uma equipe multiprofissional para melhorias em desfechos em saúde, em 2006 são criadas as residências multiprofissionais em Saúde da Mulher, considerada como pós graduação lato sensu. Nesse sentido, a equipe multiprofissional conta com a atuação de profissionais com diferentes cursos de formação inicial, sendo áreas predominantemente de ciências humanas e biológicas. A equipe multiprofissional em Saúde da Mulher pode ser composta por biomédicos, biólogos, profissionais de educação física, enfermeiros, farmacêuticos, fisioterapeutas, fonoaudiólogos e nutricionistas, por exemplo. O objetivo do programa é capacitar profissionais da área da saúde buscando atender aos princípios do SUS, em especial, a integralidade da atenção à saúde (Dantas et al., 2021) capacitando profissionais para atuarem no cuidado às mulheres na rede pública de saúde sendo mais tradicionalmente voltadas para os cursos de 
Nutrição, Farmácia, Psicologia e Educação Física.

Compreendendo a necessidade dos cuidados em saúde de maneira multi e interdisciplinar envolvendo dimensões biológicas, psicológica e sociais diversos outros cursos da área ciências da saúde e ciências humanas passaram a intervir com a população. Sabe-se que o acompanhamento nutricional juntamente com a adoção de outros hábitos saudáveis é capaz de prevenir ou erradicar várias doenças crônicas e metabólicas, promovendo benefícios diversos como melhoria na qualidade de vida, contribuindo no processo de emagrecimento (Freitas, 2017); (Maniglia, Grundmann \& da Silva, 2018).

Frente a esse cenário, o objetivo do trabalho será investigar as publicações sobre a saúde da mulher na Revista Brasileira de Obesidade, Nutrição e Emagrecimento partindo da relevância da discussão de aspectos nutricionais no que diz respeito ao cuidado integral à saúde da mulher.

\section{Metodologia}

Trata-se de um estudo de revisão retrospectiva, bibliográfica e exploratória (Ganong, 1987) realizado entre os meses de maio e junho de 2021, com abordagem descritiva, na base de dados "Revista Brasileira de Obesidade, Nutrição e Emagrecimento" através dos descritores/palavras-chave "Saúde da Mulher" "Saúde", "Mulher" e "Mulheres".

A confecção do trabalho seguiu as seguintes etapas: 1-Elaboração de pergunta norteadora; 2-Busca ou amostragem na literatura; 3-Coleta de dados; 4-Análise crítica dos estudos incluídos; 5-Discussão dos resultados; e 6-Apresentação dos resultados obtidos com o objetivo de responder à questão norteadora (Mendes, 2008); (Estrela, 2018) "Como tem sido as publicações na Revista Brasileira de Obesidade, Nutrição e Emagrecimento no que diz respeito à Saúde da Mulher?”

Os critérios de inclusão foram artigos completos publicados na revista entre o período de janeiro de 2010 a julho de 2020 identificados em junho de 2021. Somente foram incluídos artigos originais que tivessem alguma relação com a saúde da mulher no que diz a respeito à obesidade, nutrição e emagrecimento. Foram excluídos resumos (simples e expandidos) apresentados em eventos científicos e resumos (simples ou expandidos) publicados em anais de eventos científicos. Não foram incluídos debates, teses, monografia, editoriais, resenhas, resumos, TCC, dissertações, conforme apresentado na figura 1.

Posteriormente a aplicação dos critérios de inclusão e exclusão, os trabalhos foram selecionados e avaliados por título e resumo com o propósito de direcionar a temática para este estudo. Esta avaliação foi realizada por um pesquisador. Para os casos em que a leitura do resumo não fosse suficiente para definir a inclusão do artigo, foram considerados os demais critérios e a leitura na íntegra. Os dados de interesse dos trabalhos selecionados foram extraídos e registrados em planilha padronizada, que continha as principais informações sobre cada artigo.

A elegibilidade de cada estudo foi determinada pela leitura na íntegra e sua identificação é demonstrada no fluxograma da Figura 1. 
Figura 1: Fluxograma do processo de identificação e seleção de artigos.

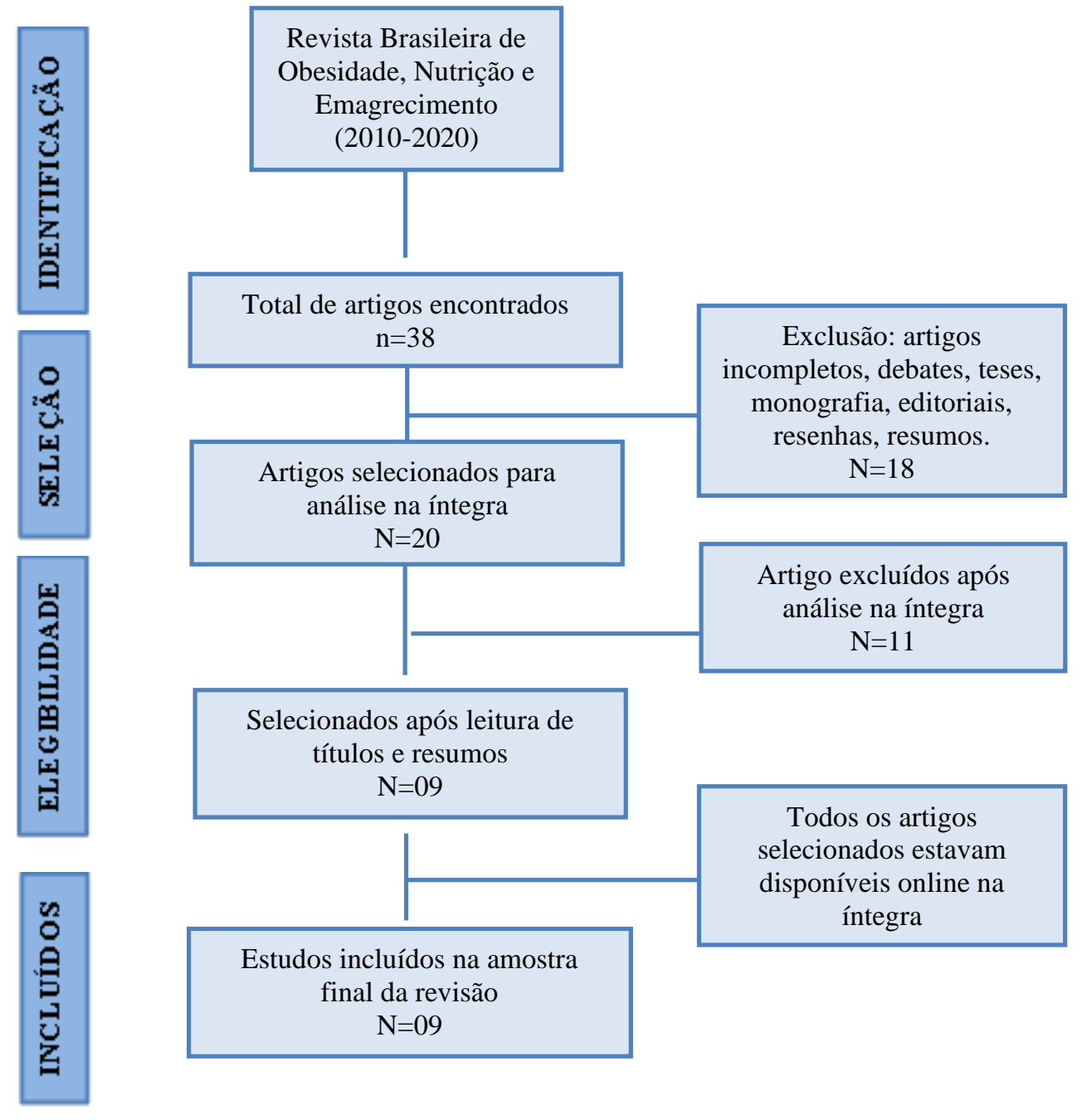

Fonte: Autores, a partir do Fluxograma PRISMA (2021).

\section{Resultados e Discussão}

Inicialmente, foram encontrados 38 artigos publicados na Revista Brasileira de Obesidade, Nutrição e Emagrecimento relacionados a temática investigada. Foram aplicados os critérios de inclusão e exclusão e selecionados 20 artigos para a análise na íntegra, no entanto, após analisados os critérios de elegibilidade, apenas 9 artigos foram aprovados para este trabalho. Todos os artigos selecionados estavam relacionados ao campo da Saúde e da Educação Física, conforme apresentado no Quadro 1. 
Quadro 1: Caracterização dos estudos incluídos na revisão segundo autores/ano de publicação, título e desenho de estudo.

\begin{tabular}{|c|c|c|c|c|}
\hline $\mathbf{N}^{\mathbf{o}}$ & Autor & Título & Ano & Metodologia \\
\hline 1 & $\begin{array}{l}\text { Hollo; Leite; } \\
\text { Navarro }\end{array}$ & $\begin{array}{l}\text { A educação nutricional como forma de } \\
\text { viabilizar o tratamento de mulheres com } \\
\text { sobrepeso e obesidade, com baixa renda, } \\
\text { atendidas numa Unidade Básica de Saúde } \\
\text { (UBS), no município de Cabreúva, SP }\end{array}$ & 2012 & Estudo de intervenção \\
\hline 2 & Braga; Quintão & $\begin{array}{l}\text { Estado nutricional e idade da menarca de } \\
\text { adolescentes de duas escolas do município de } \\
\text { Muriaé-M }\end{array}$ & 2012 & Estudo quantitativo exploratório \\
\hline 3 & $\begin{array}{l}\text { Zenatti; } \quad \text { Luz; } \\
\text { Oliveira }\end{array}$ & $\begin{array}{l}\text { Efeitos na flexibilidade e força de mulheres } \\
\text { fisicamente ativas resultantes da prática de um } \\
\text { programa de treinamento de } 2 \text { meses de Hatha } \\
\text { Yoga, modalidade Jayaprána Yoga }\end{array}$ & 2013 & $\begin{array}{l}\text { Estudo pré-experimental com } \\
\text { delineamento pré e pós-teste }\end{array}$ \\
\hline 4 & $\begin{array}{l}\text { Brugge; Mazur; } \\
\text { Cavagari }\end{array}$ & $\begin{array}{l}\text { Associação entre diagnóstico de síndrome de } \\
\text { ovários policísticos, estado nutricional e } \\
\text { consumo alimentar em mulheres em idade fértil }\end{array}$ & 2017 & Estudo transversal \\
\hline 5 & Dourado et al. & $\begin{array}{l}\text { Associação da adiposidade abdominal com } \\
\text { fatores clínicos em mulheres climatéricas. }\end{array}$ & 2018 & Estudo analítico, transversal \\
\hline 6 & $\begin{array}{l}\text { Tatsch; } \\
\text { Wichmann }\end{array}$ & $\begin{array}{l}\text { Problematizando a prática do aleitamento } \\
\text { materno em gestantes atendidas em unidades de } \\
\text { saúde. }\end{array}$ & 2018 & $\begin{array}{l}\text { Estudo transversal, quantitativo e } \\
\text { qualitativo. }\end{array}$ \\
\hline 7 & Leal et al. & $\begin{array}{l}\text { Modificações na composição corporal de } \\
\text { mulheres em risco cardiovascular pelo método } \\
\text { pilates }\end{array}$ & 2019 & Estudo descritivo e experimental \\
\hline 8 & Fonseca et al. & $\begin{array}{l}\text { Síndrome metabólica e climatério: impacto da } \\
\text { intervenção nutricional }\end{array}$ & 2020 & Estudo de intervenção \\
\hline 9 & Alahmar et al. & $\begin{array}{l}\text { Fatores associados ao estresse percebido em } \\
\text { universitários }\end{array}$ & 2020 & $\begin{array}{l}\text { Estudo observacional do tipo } \\
\text { transversal. }\end{array}$ \\
\hline
\end{tabular}

Fonte: Autores (2021).

Os principais resultados obtidos dos nove artigos selecionados foram explicitados no quadro 2, com avaliação do autor responsável de acordo com as informações levantadas.

Em relação ao ano de publicação dos artigos originais analisados, dois trabalhos $(22,22 \%)$ foram publicados em 2012 , um trabalho $(11,11 \%)$ foi publicado em 2013 , um trabalho $(11,11 \%)$ foi apresentado em 2017 , dois trabalhos $(22,22 \%)$ foram publicados em 2018, um trabalho (11,11\%) difundido em 2019 e dois (22,22\%) trabalhos divulgados em 2020.

No que se refere a metodologia, a maior prevalência encontrada foram quatro estudos de caráter transversal $(44,44 \%)$. Foram encontrados dois estudos de intervenção (22,22\%) sendo um deles observacional (11,11\%), dois estudos pré experimental com delineamento pré e pós teste $(22,22 \%)$.

De acordo com o quadro 1, todos os artigos analisados possuem relação com a área da saúde, compreendendo que a Revista Brasileira de Obesidade, Nutrição e Emagrecimento aborda estudos originais de pesquisadores predominantemente formados em Nutrição e Educação Física, o que já se era esperado, tendo em vista que essas duas áreas de concentração são as que mais tradicionalmente estão relacionadas com o desenvolvimento da obesidade, aspectos nutricionais e o processo de emagrecimento.

O Quadro 2 a seguir apresenta os principais resultados encontrados em cada um dos trabalhos analisados: 
Quadro 2: Informações dos artigos selecionados para os estudos.

\begin{tabular}{|c|c|c|c|c|}
\hline $\mathbf{N}^{0}$ & Autor/ano & Objetivo(s) & Amostra e Instrumentos & Resultados \\
\hline 1 & $\begin{array}{l}\text { Hollo; Leite; } \\
\text { Navarro } \\
2012\end{array}$ & $\begin{array}{l}\text { Adoção de hábitos } \\
\text { alimentares saudáveis, } \\
\text { visando uma redução } \\
\text { do peso corporal e } \\
\text { melhora no quadro } \\
\text { clínico. }\end{array}$ & $\begin{array}{l}\text { Foram selecionadas } 28 \\
\text { mulheres entre } 20 \text { e } 59 \text { anos, } \\
\text { com sobrepeso e obesidade e } \\
\text { comorbidades associadas, } \\
\text { numa Unidade Básica de } \\
\text { Saúde (UBS), município de } \\
\text { Cabreúva. }\end{array}$ & $\begin{array}{l}\text { Todas as mulheres apresentavam algum grau de } \\
\text { excesso de peso com maior concentração e } \\
\text { agravamento do diagnóstico na faixa etária de } 40 \\
\text { a } 59 \text { anos. Verificou-se alto consumo de óleo } \\
\text { vegetal e açúcar simples e o baixo consumo de } \\
\text { hortaliças e frutas. }\end{array}$ \\
\hline 2 & $\begin{array}{c}\text { Braga; Quintão } \\
2012\end{array}$ & $\begin{array}{l}\text { Investigar o estado } \\
\text { nutricional e sua } \\
\text { relação com a menarca. } \\
\text { Com adolescentes do } \\
\text { sexo feminino, de } 12 \text { a } \\
16 \text { anos. }\end{array}$ & $\begin{array}{l}\text { Foram avaliadas } 49 \\
\text { adolescentes de } 12 \text { a } 16 \text { anos, } \\
\text { média de } 13,8 \pm 1,14 \text { anos. }\end{array}$ & $\begin{array}{l}69,4 \% \text { das adolescentes estavam eutroficas, } \\
14,3 \% \text { com sobrepeso e } 16,3 \% \text { com obesidade. As } \\
\text { adolescentes com excesso de peso apresentaram } \\
\text { menor média e mediana de idade da menarca } \\
\text { quando comparado ao grupo das eutróficas. }\end{array}$ \\
\hline 3 & $\begin{array}{l}\text { Zenatti; Luz; } \\
\text { Oliveira } \\
2013\end{array}$ & $\begin{array}{l}\text { Expor os resultados de } \\
\text { um estudo sobre os } \\
\text { efeitos da prática de } \\
\text { Jayaprána Yoga na } \\
\text { flexibilidade e força } \\
\text { em mulheres. }\end{array}$ & $\begin{array}{l}\text { A população do estudo } \\
\text { correspondeu a } \mathrm{N}=50 \\
\text { mulheres praticantes de yoga. } \\
\text { Destas foram selecionados } \\
\text { uma amostra de } \mathrm{n}=6 \\
\text { mulheres, por atenderem aos } \\
\text { critérios pré-estabelecidos. }\end{array}$ & $\begin{array}{l}\text { Considerando tornozelo, joelho, tronco e ombro } \\
\text { ocorreram ganhos nas variáveis pesquisadas. Cabe } \\
\text { salientar que a melhora não foi mais significativa } \\
\text { provavelmente pelo fato de as pesquisadas já } \\
\text { serem praticantes de yoga. }\end{array}$ \\
\hline 4 & $\begin{array}{l}\text { Brugge; Mazur; } \\
\text { Cavagari } \\
2017\end{array}$ & $\begin{array}{l}\text { Comparar parâmetros } \\
\text { da síndrome pré- } \\
\text { menstrual (SPM) de } \\
\text { mulheres em idade } \\
\text { fértil com e sem } \\
\text { diagnóstico de SOP. }\end{array}$ & $\begin{array}{l}42 \text { universitárias, selecionadas } \\
\text { aleatoriamente, com idade } \\
\text { média observada de } 25,29 \pm \\
6,56 \text { anos. }\end{array}$ & $\begin{array}{l}\text { Observou-se maior consumo de cereais }(95,5 \%) \text {, } \\
\text { gorduras }(71,4 \%) \text { e chocolates }(52,4 \%) \text { pelas } \\
\text { participantes que apresentam a SOP e de cereais } \\
(90,5 \%) \text {, hortaliças }(66,7 \%) \text { e gorduras }(61,9 \%) \\
\text { consumidos pelas universitárias sem SOP. }\end{array}$ \\
\hline 5 & $\begin{array}{l}\text { Dourado et al. } \\
2018\end{array}$ & $\begin{array}{l}\text { Identificar a relação da } \\
\text { adiposidade abdominal } \\
\text { com fatores os clínicos } \\
\text { em mulheres } \\
\text { climatéricas assistidas } \\
\text { nas Estratégias da } \\
\text { Saúde da Família. }\end{array}$ & $\begin{array}{l}\text { População de } 30.801 \text { mulheres } \\
\text { climatéricas cadastradas nas } \\
73 \text { unidades de Estratégias da } \\
\text { Saúde da Família (ESF) de } \\
\text { Montes Claros- Minas Gerais. }\end{array}$ & $\begin{array}{l}\text { Das } 874 \text { mulheres investigadas, mostrou-se } \\
\text { associação nas alterações de RCQ com as fases do } \\
\text { climatério }(p=0,000) \text { doenças cardiovasculares } \\
(p=0,000), S M(p=0,000) \text {, aumento da pressão } \\
\text { arterial }(p=0,000) \text { e diabetes }(p=0,000) \text {. }\end{array}$ \\
\hline 6 & $\begin{array}{l}\text { Tatsch; } \\
\text { Wichmann } \\
2018\end{array}$ & $\begin{array}{l}\text { Problematizar a } \\
\text { interferência de } \\
\text { padrões socialmente } \\
\text { condicionados, como } \\
\text { crenças, mitos e tabus } \\
\text { sobre a prática do } \\
\text { aleitamento materno } \\
\text { em gestantes atendidas } \\
\text { em unidades de saúde } \\
\text { do município de Vera } \\
\text { Cruz. }\end{array}$ & $\begin{array}{l}\text { A amostra por conveniência } \\
\text { foi constituída por } 96 \\
\text { gestantes atendidas em duas } \\
\text { unidades de saúde, no período } \\
\text { de pré-natal, nos meses de } \\
\text { março a abril de } 2015 \text {. }\end{array}$ & $\begin{array}{l}\text { Referente à paridade, } 52,1 \%(\mathrm{n}=50) \text { referiram que } \\
\text { eram primigestas e } 47,9 \% \quad(\mathrm{n}=46) \text { multigestas; } \\
91,7 \% \text { (n=88) afirmaram que a prática da } \\
\text { amamentação traz benefícios para a saúde da } \\
\text { criança ou da mulher. Foram obtidos resultados } \\
\text { estatisticamente significantes entre número de } \\
\text { gestações, idade e crenças sobre o aleitamento } \\
\text { materno. }\end{array}$ \\
\hline 7 & $\begin{array}{l}\text { Leal et al. } \\
2019\end{array}$ & $\begin{array}{l}\text { Verificar os efeitos do } \\
\text { treinamento } \\
\text { supervisionado do } \\
\text { método Pilates sobre } \\
\text { composição corporal } \\
\text { de mulheres com } \\
\text { fatores de riscos } \\
\text { cardiovasculares. }\end{array}$ & $\begin{array}{l}\text { Foram recrutadas } 10 \\
\text { mulheres de meia idade, com } \\
\text { pelo menos um fatorde risco } \\
\text { cardiovascular que praticavam } \\
\text { o Pilates. Os critérios de } \\
\text { inclusão foram mulheres com } \\
\text { idade superior a } 25 \text { anos, que } \\
\text { apresentaram pelo menos um } \\
\text { dos fatores de riscos: } \\
\text { Obesidade, diabetes, } \\
\text { hipertensão, dislipidemia, } \\
\text { relação cintura quadril (RCQ) } \\
\text { de risco e que praticassem o } \\
\text { método Pilates a mais de três } \\
\text { meses, com frequência } \\
\text { mínima de duas vezes por } \\
\text { semana. }\end{array}$ & $\begin{array}{l}\text { verificou-se que houve uma redução no IMC } \\
(\mathrm{p}<0,05) \text { e na massa gorda }(\mathrm{p}<0.05) \text {, e um } \\
\text { aumento na massa livre de gordura }(\mathrm{p}<0,05) \text { e na } \\
\text { taxa metabólica }(\mathrm{p}<0,05) \text {. O tamanho do efeito } \\
\text { mostrou-se alto sobre o peso, o IMC, a massa } \\
\text { gorda, a massa livre de gordura e na taxa } \\
\text { metabólica basal. }\end{array}$ \\
\hline 8 & $\begin{array}{l}\text { Fonseca et al. } \\
\qquad 2020\end{array}$ & $\begin{array}{l}\text { Analisar o impacto da } \\
\text { intervenção nutricional } \\
\text { em mulheres com }\end{array}$ & 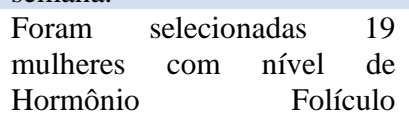 & $\begin{array}{l}\text { A adiposidade central foi a alteração com maior } \\
\text { significância estatística }(\mathrm{p}<0,0001) \text {. Observou-se } \\
\text { significativa redução no peso corporal (PC), }\end{array}$ \\
\hline
\end{tabular}


síndrome metabólica e climatérica.
Estimulante $(\mathrm{FSH})$ maior do que $40 \mathrm{mUI} / \mathrm{ml}$, compatível com a síndrome climatérica, ou cuja última menstruação (DUM) tenha ocorrido há pelo menos doze meses.

9 Alahmar et al. Avaliar o estresse percebido em 2020 universitários e este e estado nutricional, variáveis socioeconômicas demográficas.
Participaram 537 estudantes universitários $\quad(25,27 \pm 7,84$ anos) matriculados na Faculdade Barretos, entre os meses de maio de 2015 a junho de 2017. Foram coletadas informações socioeconômicas e demográficas dos participantes. Para a avaliação do estresse percebido foi utilizada a escala Perceived Stress Scale/PSS14. circunferência de cintura (CC), índice de massa corporal (IMC) e circunferência de pescoço (CP). A intervenção nutricional demonstrou que a reeducação alimentar pode ser classificada como uma das bases para o controle, e possível reversão, no quadro da Síndrome Metabólica.

A maioria era mulher $(62,7 \%)$, solteiros $(78,4 \%)$, com nível econômico B $(45,4 \%)$, residentes com os pais $(72,5 \%)$, trabalhadores $(80,7 \%)$. A prevalência foi de estudantes com excesso de peso (pré-obesidade e obesidade) (48,8\%). Dos participantes que apresentaram variação ponderal, $62,2 \%$ ganharam peso durante 0 curso de graduação. Os fatores associados ao estresse percebido em estudantes universitários foram sexo, nível econômico, uso de medicações antidepressivas e/ou ansiolíticas, performance no curso e variação de peso.

Fonte: Dados da pesquisa (2021).

O objetivo deste trabalho foi analisar os trabalhos publicados na Revista Brasileira de Obesidade, Nutrição e emagrecimento que tivessem relação com o campo da saúde da mulher no período de 2010 a 2020. No total, 9 artigos participaram do estudo.

No que se refere ao desenho da amostra, a maior prevalência encontrada foram estudos descritivos, esse dado pode ser parcialmente explicado considerando que essa abordagem metodológica que têm por objetivo determinar a distribuição de doenças e possibilita compreender as condições relacionadas à saúde, segundo o tempo, o lugar e/ou as características da população investigada. Sabe-se que a área "Saúde da Mulher" é um campo do conhecimento pertinente a diversos cursos da área da saúde, nesse sentido, estudos descritivos podem ser importantes ferramentas para o desenvolvimento de pesquisas e possíveis intervenções com essa população

Os trabalhos analisados possuíam diversas subáreas do conhecimento em saúde, apresentados por meio de estudos que tratavam aspectos epidemiológicos, sociodemográficos da atividade física nas mulheres, abordando modalidades de exercícios físicos em respostas e ajustes metabólicos. A adoção de hábitos saudáveis e a compreensão da importância dos cuidados nutricionais são variáveis que podem interferir em aspectos epidemiológicos, sociais e metabólicos que podem influenciar em desfechos na saúde da mulher.

Os resultados dos trabalhos investigados apresentam atividades preventivas no que se refere aos cuidados nutricionais que ampliam os cuidados em saúde nas mulheres, evitando possíveis prejuízos e adiando o desenvolvimento e agravo de doenças, como forma de promoção da saúde e qualidade de vida (Gutierrez, et al. 2020). Ressaltam ainda que os cuidados nutricionais em saúde da mulher devem ser promovidos através da adoção de hábitos saudáveis, como a prática regular de atividade física e boa alimentação (OMS, 2005). Destaca-se a necessidade de se compreender o conceito de saúde, considerando-a como um estado de completo bem-estar físico, mental e social, e não apenas como a ausência de doença ou enfermidade na mulher (Parhtba; Simoes, 2010).

A ampliação do entendimento da saúde da mulher como prática fundamental para a manutenção da saúde e da qualidade de vida dessa população, essencialmente as que apresentem sobrepeso ou que estejam em processo de emagrecimento, possibilita maiores questionamentos, reflexões e abordagens por parte dos pesquisadores, não apenas pesquisadores direcionados a Nutrição e a Educação Física, mas frente ao cenário multiprofissional, considerando a necessidade do cuidado de forma integral firmando a importância da prevenção e manutenção da saúde da mulher, além da possibilidade de evitar possíveis agravos de saúde ligada a outras condições (Brasil, 2006); (Warmling, et al. 2018). 
A educação nutricional aparece como alternativa de amenizar esses problemas, tendo em vista que grande parte da população não tem conhecimento do que se está ingerindo, além disso, se trata de uma alternativa com importante papel na promoção de hábitos alimentares saudáveis, principalmente na infância tendo como objetivo proporcionar os conhecimentos necessários e a motivação para formar atitudes e hábitos de alimentação saudável, adequada e variada (Rodrigues; Boog, 2006); (Rosa \& Alves, 2017).

No que tange à Nutrição e a Educação Física e Nutrição, cursos que estão mais tradicionalmente relacionados a questões nutricionais, ganho e perda de peso, a obesidade não deve ser compreendida unicamente ao sedentarismo e a falta de vontade em realizar atividade física, uma vez que existem questões relacionadas a aspectos biológicos, históricos, ecológicos, políticos, socioeconômicos, psicossociais e culturais (Souza, et al. 2018) em que o indivíduo se insere que interferem nos hábitos alimentares e na prática da atividade física/exercício físico.

Por parte dos profissionais da saúde, principalmente se tratando de saúde da mulher, é necessário que se tenha consciência das condições que interferem no peso dos indivíduos, uma vez que estão relacionadas a características particulares de cada sujeito ou grupo, enxergando a temática de forma ampliada (Azevedo, 2008); (Souza, et al. 2020).

Por fim, há de se destacar algumas limitações do presente trabalho, como o fato de não ter se buscado a temática em outras revistas e de o período da pesquisa ter ficado limitado a dez anos. No entanto, o trabalho apresenta informações que reforçam a importância dos cuidados e intervenções nutricionais em mulheres possibilitando a reflexão crítica no que diz respeito a saúde da mulher para que novas pesquisas sejam realizadas, de forma que as intervenções realizadas pelos profissionais da saúde garantam os cuidados em saúde para essa população.

\section{Considerações Finais}

Esse estudo é relevante à medida que proporciona conhecer a importância dos cuidados nutricionais no âmbito da saúde da mulher. Observa-se que a garantia dos cuidados em saúde parte do princípio multidisciplinar e que as questões quanto à alimentação podem interferir em desfechos em saúde. A adoção de hábitos saudáveis é fundamental na redução de riscos e prejuízos adversos a saúde, contribuindo na redução de problemas e agravos no que se refere a obesidade, nutrição no processo de emagrecimento, contribuindo para a manutenção da saúde e a qualidade de vida da mulher.

Os profissionais da saúde devem ter conhecimento sobre as características dessa população, no entanto para que isso ocorra é importante que exista um conhecimento específico pautado nas particularidades das mulheres, de forma que atenda às demandas desse grupo prestando uma assistência nutricional e acolhedora frente as necessidades em saúde da mulher, a fim de se reduzir prejuízos e agravos em saúde.

Destarte, torna-se excepcional a realização de novas pesquisas com a finalidade de ampliar a produção científica, compreender e promover discussões quanto a saúde da mulher no que se refere as questões nutricionais.

\section{Referências}

Azevedo, E. (2008). Reflexões sobre riscos e o papel da ciência na construção do conceito de alimentação saudável. Rev. Nutr.; 21(6):717-723.

Brasil (2004). Política nacional de atenção integral à saúde da mulher: princípios e diretrizes. Ministério da Saúde.

Brasil. (2006). Secretaria de Atenção à Saúde. Departamento de Ações Programáticas Estratégicas. Área Técnica de Saúde da Mulher. Pré-natal e puerpério: atenção qualificada e humanizada. (3a ed.).

Carvalho, S. R. (2004). As contradições da promoção à saúde em relação à produção de sujeitos e a mudança social. Cien Saude Colet; 9(3):669-678.

Carvalho, G. A saúde pública no Brasil. (2013). Estudos Avançados, 27(78), 5-26.

Cordeiro, S. N; et al. (2017). Contribuições da Psicologia à Residência Multiprofissional em Saúde da Mulher: Relato de Experiência. Rev. Polis Psique, 7, 100-115. 
Research, Society and Development, v. 10, n. 10, e561101019097, 2021

(CC BY 4.0) | ISSN 2525-3409 | DOI: http://dx.doi.org/10.33448/rsd-v10i10.19097

Czeresnia, D. (2003). O conceito de saúde e a diferença entre promoção e prevenção. In: Czeresnia D, Freitas CN. Promoção da saúde: conceitos, reflexões, tendências. FIOCRZ. 39-53.

Figueiredo, J. A. et al. (2010). Síndrome metabólica e menopausa: estudo transversal em ambulatório de ginecologia. Arq Bras Cardiol. 95(3):339-45.

Freitas, G. L. de. (2017). Discutindo a política de atenção à saúde da mulher no contexto da promoção da saúde. Revista Eletrônica De Enfermagem, 11(2).

Ganong, L.H. (1987). Integrative reviews of nursing research. Research in Nursing \& Health, 10(1), 1-11.

Gutierrez Murillo, R. S., Trujillo Rodríguez, A. D. P., Cardozo Prada, Y. L., Mosquera Conde, O. L., Pino Parra, K. Y., \& Negri, E. D. (2020). Violência no namoro como questão de saúde pública: experiências de (des)construção de estigmas sociais em ambiente universitário. Research, Society and Development, 9(7), e290972879.

Goes, E. F., Nascimento, E. R. (2013). Mulheres negras e brancas e os níveis de acesso aos serviços preventivos de saúde: uma análise sobre a desigualdade. Saúde Debate. 37(99):571-9.

Maniglia, F. P., Grundmann, C. G., \& da Silva, T. A. (2018). Relação entre características alimentares e composição corporal com a qualidade de vida de mulheres submetidas à cirurgia bariátrica. RBONE - Revista Brasileira De Obesidade, Nutrição E Emagrecimento, 12(69), 76-83.

Martins, A. L. (2001). Mortalidade materna: maior risco para as mulheres negras no Brasil. Jornal da Rede, Rede Saúde.

Mendes, K. D., Silveira, R. C. C. \& Galvão, C. M. (2008). Revisão integrativa: método de pesquisa para a incorporação de evidências na saúde e na enfermagem. Texto \& Contexto - Enfermagem, 17(4), 758-764.

Mori, M. E., Coelho, V. L. D., \& Estrella, R. C. N. (2006). Sistema Único de Saúde e políticas públicas: atendimento psicológico à mulher na menopausa no Distrito Federal, Brasil. Cad. Saúde Pública. ;22(9):1825-33.

Rodrigues, E. M.; \& Boog, M. C. F. (2006). Problematização como estratégia de educação nutricional com adolescentes obesos. Cadernos de Saúde Pública [online], 22923-931.

Souza, V. A. de, Machado, G. N., Arrué, A. M., Luzardo, A. R., Jantsch, L. B., \& Danski, M. T. R. (2020). As Práticas Integrativas e Complementares na atenção à saúde da mulher. Research, Society and Development, 9(8), e81985379.

Souza, Y. P., Bezerra, A. M., Fabrício, N. P., Tavares, N. B. F., Félix, N. D. C., Viana, M. C. A., et al. (2018). A qualidade de vida de idosos com obesidade ou sobrepeso. Rev Bras Ciênc Saúde [serial on the internet] 22(2):155-64.

Rosa, Q. P. P., \& Alves, M. K. (2017). Prevalência e fatores associados ao excesso de peso em mulheres adultas colaboradoras de uma unidade de alimentação e nutrição. RBONE - Revista Brasileira De Obesidade, Nutrição E Emagrecimento, 11(66), 428-436.

Rossi, S. S. (1987). A constituição do sistema de saúde no Brasil. São Paulo, 1980. Cópia mimeografada utilizada no Curso de Política de Saúde promovido pela Plenária de Entidades de Saúde, 70-77.

Veras, R. (2007). Fórum. Envelhecimento populacional e as informações de saúde do PNAD: demandas e desafios contemporâneos. Introdução. Cadernos de Saúde Pública [online]. 23, 2463-2466.

Warmling, C. M., Fajardo, A. P., Meyer, D. E. \& Bedos, C. (2018). Práticas sociais de medicalização \& humanização no cuidado de mulheres na gestação. Cadernos de Saúde Pública, 34(4), 1-11. 